\title{
A qualidade em atividades de grupo de fitness: construção e validação do questionário "Qualidade do Instrutor de Fitness - Atividades de Grupo" (QIF-AG)
}

\author{
Francisco Ascenso Campos ${ }^{1}$, Vera Costa Simões ${ }^{2}$, Susana Alves Franco ${ }^{2}$ \\ ${ }^{1}$ Escola Superior de Educação - Instituto Politécnico de Coimbra \\ ${ }^{2}$ Escola Superior de Desporto de Rio Maior - Instituto Politécnico de Santarém
}

\begin{abstract}
Resumo: Sabendo que comportamentos inadequados ou não gostar do instrutor são motivos invocados pelos praticantes de atividades de grupo de fitness para abandonar a prática, é cada vez mais recomendado aos ginásios implementar estratégias para diminuir o abandono e aumentar a fidelização dos praticantes (Franco, Pereira, \& Simões, 2008). Pelo referido, é objetivo deste trabalho construir e validar um questionário que permita avaliar a qualidade do instrutor em atividades de grupo de fitness. A construção teve por base um modelo teórico constituído por 4 dimensões e 25 categorias (Campos, 2015). Após cumprimento das várias fases subjacentes a este processo, nomeadamente após análise fatorial exploratória e confirmatória, o questionário QIF-AG é considerado válido, apesar de com algumas limitações, apresentando uma estrutura fatorial com 2 fatores correlacionados: Qualidade Relacional (11 itens) e Qualidade Técnico-pedagógica (14 itens).
\end{abstract}

Palavras-chave: Fitness; Instrutor; QIF-AG; Qualidade.

The quality in fitness group activities: construction and validation of the questionnaire "Fitness Instructor Quality - Group Activities" (QIF-AG): Knowing that inadequate behaviors or don't like of the instructor are reasons presented by group fitness participants to dropout, it is increasingly recommended to gyms to apply strategies do decrease the dropout and increase the loyalty of participants (Franco, Pereira, \& Simões, 2008). By that, the aim of this study is to construct and validate a questionnaire to assess the quality of the group fitness instructor. The construction was based on a theoretical model with 4 dimensions and 25 categories (Campos, 2015). After completing of the several underlying phases of this process, in particular after the exploratory and confirmatory factor analysis, the questionnaire QIF-AG is considered valid, although with some limitations, presenting a factorial structure with 2 correlated factors: Relational Quality (11 items) and Technical-pedagogical Quality (14 items).

Keywords: Fitness; Instructor; QIF-AG; Quality.

A qualidade da experiência tida com um determinado serviço, no caso de proporcionar satisfação, pode provocar intenção de fidelização em consequência dessa vivência agradável (Baker \& Crompton, 2000). É possível verificar que esta relação está presente nos serviços na área do fitness pois a vivência de um serviço de qualidade pode fazer com que os indivíduos que o utilizam se sintam mais satisfeitos (Fernandéz, Carrion, \& Ruíz, 2012) e se mantenham fidelizados por mais tempo (Pedragosa \& Correia, 2009).

Relativamente às atividades de grupo (um dos serviços oferecidos na área), o instrutor (responsável pela prestação direta do serviço), pela sua baixa ou elevada qualidade, pode ter um papel preponderante na satisfação e fidelização dos praticantes. Esta relação é estudada em Fernandéz et al. (2012), Murray e Howat (2002), Nuviala, Pérez-Ordás, Osuna, Grao-Cruces, Nuviala, e Jurado (2012), e Papadimitriou e Karteroliotis (2000). Comportamentos inadequados (Francis \& Seibert, 2000) e/ou não gostar do instrutor (Franco, Pereira, \& Simões, 2008), entre outros, são alguns dos motivos invocados pelos praticantes para o abandono da prática. Pelo anteriormente referido, é cada vez mais recomendado aos ginásios implementar estratégias para diminuir o abandono e/ou aumentar a fidelização (Franco et al., 2008), pelo que o estudo dos indicadores associados à qualidade do instrutor é adequado e pertinente.

Campos (2015), com o objetivo de identificar e definir os indicadores de qualidade dos instrutores de atividades de grupo de fitness, considerando a opinião de diferentes intervenientes (praticantes, instrutores, formadores, diretores técnicos e proprietários/gestores de ginásios) para uma caracterização

${ }^{1}$ Dados de contacto para correspondência: Francisco José Ascenso Campos, Rua Dom João III, Solum, 3030-329 Coimbra, Portugal. E-mail: francicampos@esec.pt. 
mais abrangente, aplicou 100 entrevistas a esses diferentes intervenientes. Todas as entrevistas foram analisadas através da técnica análise de conteúdo e, considerando igualmente as sugestões dos vários painéis de experts e a revisão da literatura, foram listados, definidos e agrupados de acordo com dimensões mais abrangentes (Profissional, Relacional, Técnica, e Pedagógica), os indicadores de qualidade associados ao instrutor de atividades de grupo de fitness (Campos, 2015), apresentados na tabela 1.

Tabela 1. Indicadores de qualidade do instrutor de atividades de grupo de fitness

\begin{tabular}{|c|c|c|}
\hline Dimensão & Categoria & \\
\hline \multirow{6}{*}{ Profissional } & Assiduidade & Um profissional que não falta às aulas e/ou aos compromissos assumidos. \\
\hline & Dedicação & $\begin{array}{l}\text { Um profissional empenhado e voluntarioso, que demonstra vontade, gosto e entrega no } \\
\text { que faz. }\end{array}$ \\
\hline & Ética & $\begin{array}{l}\text { Um profissional correto, que respeita os outros, e que profissionalmente nutre uma } \\
\text { relação sã com os praticantes e com a restante comunidade. }\end{array}$ \\
\hline & Experiência & $\begin{array}{l}\text { Um profissional que já exerce há algum tempo, e de forma continuada, atividade } \\
\text { profissional como instrutor de atividades de grupo de fitness. }\end{array}$ \\
\hline & Imagem & $\begin{array}{l}\text { Um profissional com uma aparência agradável, e boa apresentação, nomeadamente em } \\
\text { termos de higiene e/ou vestuário utilizado, adequada ao contexto e às características dos } \\
\text { praticantes. }\end{array}$ \\
\hline & Pontualidade & $\begin{array}{l}\text { Um profissional que chega a horas, ou mesmo antes, para preparar o material necessário, } \\
\text { confirmar se está tudo em conformidade, e/ou receber os praticantes. }\end{array}$ \\
\hline \multirow{8}{*}{ Relacional } & Boa Disposição & Uma pessoa alegre, divertida, sorridente e com sentido de humor. \\
\hline & Comunicação & $\begin{array}{l}\text { Uma pessoa que utiliza uma linguagem adequada, clara, objetiva e sucinta, fazendo-se } \\
\text { entender, e que é assertivo na informação prestada. }\end{array}$ \\
\hline & Cordialidade & Uma pessoa educada, cortês e com "boas maneiras" para os praticantes. \\
\hline & Disponibilidade & $\begin{array}{l}\text { Uma pessoa acessível e disponível para os praticantes, para problemas, dúvidas e/ou } \\
\text { questões colocadas. }\end{array}$ \\
\hline & Empatia & $\begin{array}{l}\text { Uma pessoa que mantém uma relação de afinidade, proximidade e cumplicidade com os } \\
\text { praticantes. }\end{array}$ \\
\hline & Honestidade & Uma pessoa sincera, franca e frontal na sua abordagem. \\
\hline & Humildade & $\begin{array}{l}\text { Uma pessoa modesta, que não se vangloria, que admite poder sempre aprender algo } \\
\text { mais, mesmo com os outros, e que tem a capacidade de aceitar a crítica e retificar o seu } \\
\text { comportamento. }\end{array}$ \\
\hline & Simpatia & $\begin{array}{l}\text { Uma pessoa amável, afável e acolhedora, que cumprimenta sempre os praticantes, } \\
\text { mesmo quando os encontra em outro contexto. }\end{array}$ \\
\hline \multirow{7}{*}{ Técnica } & Condição Física & Um técnico que se encontra em "boa forma", com um adequado nível de condição física. \\
\hline & Conhecimento & $\begin{array}{l}\text { Um técnico com conhecimento específico na área do fitness e em geral na área do } \\
\text { desporto. }\end{array}$ \\
\hline & $\begin{array}{l}\text { Domínio } \\
\text { Musical }\end{array}$ & $\begin{array}{l}\text { Um técnico com sentido rítmico, que domina a música e as suas contagens, que respeita a } \\
\text { estrutura musical, articulando o exercício com a cadência musical. }\end{array}$ \\
\hline & $\begin{array}{l}\text { Execução } \\
\text { Técnica }\end{array}$ & $\begin{array}{l}\text { Um técnico que, em termos gerais, é um bom executante, realizando os exercícios em } \\
\text { termos técnicos e posturais de forma correta. }\end{array}$ \\
\hline & Formação & $\begin{array}{l}\text { Um técnico com formação na área, específica do fitness e genérica das ciências do } \\
\text { desporto, seja académica ou profissional, certificada, contínua e/ou credível. }\end{array}$ \\
\hline & Inovação & $\begin{array}{l}\text { Um técnico que inova, sendo para tal original e criativo, diversificando as aulas de forma } \\
\text { a não as tornar repetitivas e monótonas. }\end{array}$ \\
\hline & Planeamento & $\begin{array}{l}\text { Um técnico que planeia as suas aulas, e o faz de um modo metódico, demonstrando tê-las } \\
\text { sempre bem preparadas e organizadas, considerando as características dos praticantes. }\end{array}$ \\
\hline \multirow{4}{*}{ Pedagógica } & Adequabilidade & $\begin{array}{l}\text { Um técnico que adequa a sua intervenção de acordo com as condicionantes da aula, que } \\
\text { por vezes tem de ajustar e improvisar, sendo para tal flexível e versátil. }\end{array}$ \\
\hline & Dinamismo & Um técnico dinâmico, enérgico, ativo, extrovertido e expansivo na sua intervenção. \\
\hline & Instrução & $\begin{array}{l}\text { Um técnico que instrui de forma correta, adequada e pertinente, e que observa a } \\
\text { execução motora e corrige sempre que necessário. }\end{array}$ \\
\hline & Motivação & $\begin{array}{l}\text { Um técnico que cativa, elogia, encoraja e incentiva os praticantes, motivando-os para a } \\
\text { prática, criando um clima de aula positivo. }\end{array}$ \\
\hline
\end{tabular}


Com este trabalho, denominado por estudo preliminar em Hill e Hill (2002), pretende-se, através da especificação das variáveis a investigar, elaborar um questionário de avaliação da qualidade do instrutor, no âmbito das atividades de grupo de fitness. 0 objetivo da presente investigação é construir e validar um questionário que permita avaliar a qualidade do instrutor de atividades de grupo, considerando a perceção dos praticantes de fitness, tendo por base as categorias e respetivas dimensões apresentadas no estudo de Campos (2015).

\section{MÉTODO}

\section{Procedimentos}

Considerando os objetivos definidos, foram seguidas as recomendações de vários autores de referência relativamente à construção de questionários (Almeida \& Freire, 2003; DeVellis, 2003; Gillham, 2003; Hill \& Hill, 2002; Moreira, 2009).

No estudo preliminar 1, após a elaboração do modelo prévio contendo os indicadores referenciados na literatura em relação à qualidade do instrutor de atividades de grupo, foram aplicadas 100 entrevistas a diferentes intervenientes na área do fitness (proprietários/gestores, diretores técnicos, formadores, instrutores de atividades de grupo e praticantes de atividades de grupo), objetivando recolher mais informação acerca do construto em análise. Através da análise de conteúdo foram definidos 25 indicadores (categorias), os quais foram agrupados em 4 áreas distintas (dimensões), sendo assim definidas as variáveis a incluir na primeira versão do questionário. Para tal, foram redigidas 150 questões, 6 para cada uma das 25 categorias (fase 1), seguindo o recomendado em Hill e Hill (2002).

Na fase 2 - estudo preliminar 2, ou pré-teste - foi efetuada uma primeira aplicação do questionário com objetivo de verificar a adequação dos itens e escala de resposta, cumprindo as sub-fases recomendadas por Hill e Hill (2002). 0 questionário foi aplicado pessoalmente pelo investigador a uma amostra pequena ( $n=60$, subdividida em 10 grupos de 6 praticantes), onde foi explicado o âmbito e objetivo do estudo. Imediatamente a seguir ao preenchimento, em cada grupo, os inquiridos foram convidados a falar sobre qualquer problema, dúvida ou dificuldade aquando do seu preenchimento e, após troca de impressões, foram recolhidos os questionários para posterior análise. Foi efetuada uma análise aos resultados do questionário com o objetivo de verificar quais as questões que não foram respondidas (por serem ambíguas ou sensíveis por solicitarem informação pessoal e/ou desconhecida), e examinar a distribuição das respostas em cada pergunta (alguns motivos que podem reduzir a variabilidade da resposta são as perguntas que convidam a uma resposta socialmente aceitável e as com possibilidade de resposta alternativa restrita ou inadequada).

Na fase 3 - revisão por experts -, recomendada por Almeida e Freire (2002) e Hill e Hill (2002), entre outros, a primeira versão do questionário foi enviada a um painel de seis experts, doutorados em Ciências do Desporto e com experiência na área do fitness, com o objetivo de avaliar a adequabilidade dos itens em relação ao que efetivamente se pretendia medir, e a sua facilidade de compreensão. Foram fornecidas indicações no sentido de alterar a formulação de alguns itens, tal como eliminar outros (por serem bastante idênticos a outros itens da mesma categoria, e mantendo para todas as categorias um mínimo de 3 itens associados), pelo que o questionário foi revisto e reformulado segundo as considerações e recomendações de ambos os grupos consultados nas fases 2 (revisão por praticantes) e 3 (revisão por experts).

Na fase 4 - estudo piloto -, a versão 2 do questionário foi aplicada a uma amostra de 100 praticantes de atividades de grupo de fitness, conforme o recomendado em Hill e Hill (2002), com objetivo de realizar uma primeira análise estatística, e desta forma ter uma primeira noção relativamente à qualidade psicométrica do questionário. Para além dos valores de consistência interna, via alfa de cronbach, foram analisados os resultados dos valores de correlação entre itens (item-item) e entre itens e escala (item-total), para refletir sobre a necessidade de eliminação ou alteração de alguns itens. Após esta fase foram eliminados 4 itens, passando a nova versão do questionário (versão 3) a ser constituída por 88 itens.

Na fase 5 - análise fatorial exploratória -, o questionário foi aplicado a uma nova amostra de 450 praticantes de atividades de grupo. Foram analisados os índices de ajustamento da sua estrutura fatorial, comparando-os com os índices indicados na literatura de referência, que nos permitem aceitar ou alterar a estrutura fatorial para que a mesma possa ser considerada válida. Após nova revisão do questionário foi elaborada uma nova versão (versão 4), com apenas 25 itens (os que apresentavam maiores valores de correlação com a escala), a qual foi submetida a análise fatorial confirmatória.

Na fase 6 - análise fatorial confirmatória -, a versão 4 do questionário foi aplicada a uma nova amostra (960 praticantes). Tal como na análise fatorial exploratória, foram analisados os índices de ajustamento da sua estrutura fatorial comparando-os com os índices de ajustamento indicados na literatura de referência. 


\section{Participantes}

Considerando as diferentes fases do processo de construção e validação do instrumento (Hill \& Hill, 2002), participaram no estudo quatro amostras distintas:

a) No estudo preliminar 2, ou pré-teste, participaram 60 praticantes de atividades de grupo de fitness (24.3 \pm 2.6 anos), 25 do género masculino e 35 do género feminino;

b) No estudo piloto, para $n=100(21.2 \pm 3.1$ anos), 53 praticantes são do género masculino e 47 do género feminino;

c) Na análise fatorial exploratória, para um $n=450$ (38.1 \pm 16.4 anos), 110 praticantes são do género masculino e 340 do género feminino;

d) Em relação à análise fatorial confirmatória, participaram 960 praticantes de atividades de grupo (34.8 \pm 15.3 anos), 250 do género masculino e 710 do género feminino.

\section{Análise dos dados}

O grau de confiança que temos na informação obtida é avaliado através da fiabilidade, via consistência interna, pois fornece coeficientes superiores à estabilidade temporal, explicado pelo facto de os dados não sofrerem influência de circunstâncias quotidianas que determinam oscilações normais na aplicação de um questionário (Almeida \& Freire 2003). 0 coeficiente de consistência interna de 0.80 ou mais é bom e o coeficiente de 0.70 a 0.80 é aceitável (Hill \& Hill, 2002; Pestana \& Gageiro, 2008). Em alguns estudos admitem-se valores de consistência interna de 0.60 a 0.70 , o que, segundo a bibliografia, é fraco. A consistência interna é avaliada no estudo piloto, análise fatorial exploratória e confirmatória, utilizando o software SPSS 21.

A análise fatorial exploratória, via análise fatorial das componentes principais, pretende identificar um conjunto menor de variáveis hipotéticas (fatores) com o objetivo de reduzir a dimensão dos dados sem perda de informação (Pereira, 2004). Objetiva igualmente postular um modelo que correlacione as variáveis observáveis (itens), pressupondo existir um menor número de variáveis não observáveis (fatores) que expressam o que existe de comum nas variáveis originais (Hill \& Hill 2002; Maroco, 2007; Pestana \& Gageiro, 2008; Pett et al., 2003). Para realizar a análise fatorial exploratória (fase 5) as variáveis foram submetidas a análise fatorial das componentes principais, sendo a estrutura fatorial proposta definida com base nos critérios indicados na literatura (Hill \& Hill 2002; Maroco, 2007; 2010; Moreira, 2009; Munro, 2005; Pereira, 2004; Pestana \& Gageiro, 2008):

a) Força da relação existente entre as variáveis (itens) superior a 0.6, mensurada através da aplicação dos testes de Kaiser-Meyer-Olkin (KMO) e esfericidade de Bartlett;

b) Comunalidades extraídas, representando a percentagem da variância comum entre variáveis (itens) e fatores extraídos, superiores a 32\%;

c) Percentagem total da variância comum que é explicada pela solução fatorial superior a $40 \%$ ou com eigenvalue (valor próprio) igual ou superior a 1 ;

d) Peso fatorial, via correlação da variável (item) com o fator, igual ou superior a 0.4. Para realizar a análise fatorial exploratória, foi utilizado o software SPSS 21.

Relativamente à análise fatorial confirmatória, foi utilizada a técnica Structural Equation Modeling (SEM) e o método de estimação da máxima verosimilhança Maximum Likelihood Estimation (MLE). Foram analisados os dados de cada um dos fatores, separadamente, e da estrutura fatorial global, para verificar qual o modelo que apresenta melhores índices (estrutura com 2 fatores independentes ou com 2 fatores correlacionados). Os modelos propostos foram avaliados de acordo com os indicadores de bondade de ajustamento e respetivos testes recomendados na literatura (Blunch, 2008; Bryant \& Yarnold, 2000; Byrne, 2001; Maroco, 2007; Moreira, 2009; Munro, 2005):

a) Chi-Square $\left(X^{2}\right)$ (diferença entre as matrizes de covariância observadas e esperadas, não ajustada aos graus de liberdade) superior a 0.05 ;

b) Root Mean Square Error Aproximation (RMSEA) (ajustamento global do modelo relativamente aos graus de liberdade) inferior a 0.05 (perfeito) ou 0.08 (aceitável);

c) Normed of Fit Index (NFI) (comparação do modelo proposto com o modelo nulo, não ajustado aos graus de liberdade) superior a 0.80 ;

d) Comparative Fit Index (CFI) (índice de adequação global do modelo) superior a 0.90. Para realizar a análise fatorial confirmatória, foi utilizado o software AMOS 21.

\section{RESULTADOS}

Após realização de várias análises conjugando os itens de diferentes formas, para obter a estrutura com melhores índices, foram obtidos resultados que permitem aceitar uma estrutura fatorial com as 25 categorias do modelo teórico apresentado por Campos (2015).

0 valor da medida de adequação da amostra (MAA) de Kaiser-Meyer-Olkin (KMO) é 0.969, o que 
permite concluir sobre a possibilidade de uma análise fatorial dos dados com qualidade muito boa. Relativamente ao teste da esfericidade de Bartlett, é possível rejeitar a hipótese nula de não existir correlação entre as variáveis. (Pestana \& Gageiro, 2008). Em todas as variáveis os valores das comunalidades são superiores a 32\% (Tabachnik \& Fidell, 2006). Para determinar o número de fatores a reter utilizou-se o critério de Kaiser, sendo retidos 2 fatores (explicam 63\% da variância total observada nas 25 variáveis extraídas). Pela análise da matriz dos componentes, após a aplicação do método de rotação varimax (tabela 2), é possível verificar que as saturações das variáveis em cada fator são todas superiores ao mínimo (40\%), pelo que é possível aceitar a estrutura fatorial. Esta é constituída por 2 fatores, um com 11 variáveis e outro com 14, perfazendo um total de 25 variáveis.

Tabela 2. Matriz dos componentes após rotação varimax

\begin{tabular}{|c|c|c|}
\hline Item & Fator 1 & Fator 2 \\
\hline 37. É uma pessoa simpática. & 0.896 & \\
\hline 31. É uma pessoa divertida. & 0.869 & \\
\hline 75. Tem uma relação de proximidade com os praticantes. & 0.814 & \\
\hline 47. Tem uma relação saudável com os praticantes. & 0.788 & \\
\hline 16. Demonstra disponibilidade para ouvir qualquer problema que possa surgir. & 0.723 & \\
\hline 58. É uma pessoa com "boas maneiras". & 0.688 & \\
\hline 35. Demonstra ser uma pessoa sincera. & 0.653 & \\
\hline 71. Encoraja os praticantes durante a prática. & 0.636 & \\
\hline 53. Demonstra ter cuidado relativamente à sua imagem. & 0.602 & \\
\hline 7. Fala de uma forma clara. & 0.579 & \\
\hline 36. Demonstra capacidade para aceitar uma crítica. & 0.555 & \\
\hline 64. Demonstra respeitar o ritmo da música. & & 0.776 \\
\hline 86. Executa bem os exercícios em termos técnicos. & & 0.753 \\
\hline 63. Demonstra ter conhecimento geral na área do desporto. & & 0.742 \\
\hline 74. Não falta às aulas marcadas. & & 0.707 \\
\hline 73. Demonstra entrega em relação ao que faz. & & 0.702 \\
\hline 52. Demonstra já trabalhar na área do fitness há algum tempo. & & 0.694 \\
\hline 25. Demonstra ter formação específica na área do fitness. & & 0.685 \\
\hline 22. Perante algum imprevisto, tem a capacidade de adequar a aula. & & 0.651 \\
\hline 26. Demonstra ter a aula bem organizada. & & 0.639 \\
\hline 54. Chega atempadamente à aula. & & 0.618 \\
\hline 66. É original nas aulas que apresenta. & & 0.615 \\
\hline 24. Está atento ao desempenho dos praticantes quando fazem um exercício. & & 0.474 \\
\hline 38. Demonstra ter uma boa condição física. & & 0.471 \\
\hline 87. É extrovertido na sua intervenção. & & 0.469 \\
\hline
\end{tabular}

Nota. Método de rotação varimax com normalização de Kaiser. Rotação convergiu em 3 interações.

Para terminar a análise fatorial exploratória, foi analisada a consistência interna via alfa de cronbach, sendo obtidos os seguintes resultados: escala total (25 itens) - 0.967; fator 1 (11 itens) - 0.931; fator 2 (14 itens) - 0.953. Os resultados indicam, de acordo com Hill e Hill (2002) e Pestana e Gageiro (2008), valores de consistência interna muito boa, no entanto, os valores obtidos na escala total (0.967) e no fator 2 (0.953) remetem-nos para a possível redundância e fraca discriminação dos itens pelos valores de alta fiabilidade $(>0.95)$.

Após aceitação desta estrutura fatorial, constituída por 25 itens agrupados em 2 fatores (Qualidade Relacional e Qualidade Técnico-pedagógica), foi elaborada a versão 4 do QIF-AG. Importa clarificar que cada um dos itens está associado a uma das 25 categorias do modelo proposto no estudo preliminar (Campos, 2015). Na tabela 3 apresentamos os 25 itens e as categorias (fatores) às quais estão associados. Esta nova estrutura fatorial (versão 4 do questionário QIF-AG) serviu de base para realização da análise fatorial confirmatória.

Em termos de análise fatorial confirmatória, no fator Qualidade Relacional existe validade convergente pois as saturações fatoriais são elevadas (0.75) e significativas $(t>1.96)$. A fiabilidade verifica-se, resultante da averiguação da consistência interna (0.93) e fiabilidade composta (0.99), e a variância extraída (0.56) é aceitável pelo seu valor superior a 0.5. Em relação à possível existência de normalidade, aferida pelo método da máxima verosimilhança $M L E$ (tabela 4), os valores referentes aos diversos rácios críticos de achatamento estão fora do intervalo +2 e -2 , indicando a não existência de normalidade (Arbuckle \& Wothke, 1999), pelo que a distribuição das variáveis apresentam desvios em relação à curva normal. 0 rácio crítico de assimetria excede o valor absoluto 2 revelando que as variáveis não apresentam distribuição simétrica. Neste caso também não se verifica a existência de normalidade 
multivariante, entre o conjunto de variáveis considerado, pois o valor do último indicador também apresenta um valor fora do intervalo entre +2 e -2 .

Tabela 3. Dimensões, categorias e itens da versão 4 do QIF-AG

\begin{tabular}{ll}
\hline Item & Categoria \\
\hline Qualidade Relacional & \\
1. Fala de uma forma clara. & Comunicação \\
2. Demonstra disponibilidade para ouvir qualquer problema que possa surgir. & Disponibilidade \\
7. É uma pessoa divertida. & Boa Disposição \\
8. Demonstra ser uma pessoa sincera. & Honestidade \\
9. Demonstra capacidade para aceitar uma crítica. & Humildade \\
10. É uma pessoa simpática. & Simpatia \\
12. Tem uma relação saudável com os praticantes. & Ética \\
14. Demonstra ter cuidado relativamente à sua imagem. & Imagem \\
16. É uma pessoa com "boas maneiras". & Cordialidade \\
20. Encoraja os praticantes durante a prática. & Motivação \\
23. Tem uma relação de proximidade com os praticantes. & Empatia \\
Qualidade Técnico-pedagógica & \\
3. Perante algum imprevisto, tem a capacidade de adequar a aula. & Adequabilidade \\
4. Está atento ao desempenho dos praticantes quando fazem um exercício. & Instrução \\
5. Demonstra ter formação específica na área do fitness. & Formação \\
6. Demonstra ter a aula bem organizada. & Planeamento \\
11. Demonstra ter uma boa condição física. & Condição Física \\
13. Demonstra já trabalhar na área do fitness há algum tempo. & Experiência \\
15. Chega atempadamente à aula. & Pontualidade \\
17. Demonstra ter conhecimento geral na área do desporto. & Conhecimento \\
18. Demonstra respeitar o ritmo da música. & Domínio Musical \\
19. É original nas aulas que apresenta. & Inovação \\
21. Demonstra entrega em relação ao que faz. & Dedicação \\
22. Não falta às aulas marcadas. & Assiduidade \\
24. Executa bem os exercícios em termos técnicos. & Execução Técnica \\
25. É extrovertido na sua intervenção. & Dinamismo \\
\hline
\end{tabular}

Tabela 4. Valores de normalidade: Qualidade Relacional

\begin{tabular}{lcccccc}
\hline Variável & Mínimo & Máximo & Assimetria & Rácio Crítico & Achatamento & Rácio Crítico \\
\hline Q23 & 1.000 & 7.000 & -1.387 & -17.543 & 2.097 & 13.263 \\
Q20 & 2.000 & 7.000 & -1.545 & -19.546 & 3.016 & 19.076 \\
Q16 & 3.000 & 7.000 & -1.305 & -16.512 & 1.164 & 7.359 \\
Q14 & 1.000 & 7.000 & -1.551 & -19.618 & 3.647 & 23.064 \\
Q12 & 3.000 & 7.000 & -1.393 & -17.623 & 1.654 & 10.459 \\
Q10 & 1.000 & 7.000 & -1.720 & -21.751 & 3.221 & 20.368 \\
Q9 & 1.000 & 7.000 & -1.116 & -14.114 & 0.926 & 5.854 \\
Q8 & 2.000 & 7.000 & -1.303 & -16.475 & 1.292 & 8.171 \\
Q7 & 2.000 & 7.000 & -1.544 & -19.529 & 2.367 & 14.969 \\
Q2 & 2.000 & 7.000 & -1.317 & -16.655 & 1.552 & 9.813 \\
Q1 & 2.000 & 7.000 & -1.279 & -16.175 & 1.677 & 10.603 \\
\hline
\end{tabular}

No que concerne aos índices de ajustamento, os valores indicam um bom ajustamento global do modelo aos dados recolhidos se tivermos em consideração o CFI (0.953) e o NFI (0.946). Não indicam um bom ajustamento se considerarmos o $X^{2}$ (353.294; $\left.p<0.001\right)$ e o RMSEA (0.086), sendo o primeiro valor afetado pelo tamanho da amostra e pelo não cumprimento do pressuposto da normalidade. Foram igualmente analisados os efeitos diretos entre as variáveis e o fator, através da estimativa estandardizada dos valores, sendo possível verificar que todas as variáveis apresentam uma relação positiva e significativa bastante elevada com o fator.

Para o fator Qualidade Técnico-pedagógica, tal como na Qualidade Relacional, existe validade convergente pois as saturações fatoriais são elevadas (0.71) e significativas $(t>1.96)$. A fiabilidade 
verifica-se, resultante da averiguação da consistência interna (0.93) e fiabilidade composta (0.99), e a variância extraída (0.50) é igualmente aceitável. Em termos de existência de normalidade, os valores são idênticos aos obtidos na dimensão Qualidade Relacional (tabela 5), sendo indicativos da não existência de normalidade (Arbuckle \& Wothke, 1999).

Tabela 5. Valores de normalidade: Qualidade Técnico-pedagógica

\begin{tabular}{lcccccc}
\hline Variável & Mínimo & Máximo & Assimetria & Rácio Crítico & Achatamento & Rácio Crítico \\
\hline Q25 & 2.000 & 7.000 & -1.494 & -18.901 & 2.393 & 15.132 \\
Q24 & 2.000 & 7.000 & -1.573 & -19.899 & 2.615 & 16.538 \\
Q22 & 2.000 & 7.000 & -2.181 & -27.583 & 6.274 & 39.680 \\
Q21 & 2.000 & 7.000 & -1.400 & -17.704 & 2.057 & 13.008 \\
Q19 & 3.000 & 7.000 & -1.018 & -12.874 & 0.393 & 2.484 \\
Q18 & 1.000 & 7.000 & -1.645 & -20.804 & 2.949 & 18.652 \\
Q17 & 2.000 & 8.000 & -1.403 & -17.746 & 1.957 & 12.379 \\
Q15 & 1.000 & 7.000 & -1.509 & -19.089 & 3.185 & 20.144 \\
Q13 & 2.000 & 7.000 & -1.109 & -14.031 & 0.766 & 4.846 \\
Q11 & 2.000 & 7.000 & -1.453 & -18.378 & 2.193 & 13.869 \\
Q6 & 1.000 & 7.000 & -1.487 & -18.808 & 3.299 & 20.867 \\
Q5 & 3.000 & 7.000 & -1.242 & -15.709 & 1.006 & 6.365 \\
Q4 & 2.000 & 7.000 & -1.222 & -15.452 & 1.916 & 12.120 \\
Q3 & 2.000 & 7.000 & -1.171 & -14.814 & 1.547 & 9.781 \\
\hline
\end{tabular}

As medidas indicam um bom ajustamento global do modelo aos dados se considerarmos o CFI (0.938), RMSEA (0.078) e NFI (0.929). No entanto, não indica um bom ajustamento se considerarmos o $X^{2}$ (526.630; $p<0.001$ ), sendo este valor afetado pelo tamanho da amostra e pelo não cumprimento do pressuposto da normalidade. Ao analisar os efeitos diretos entre variáveis e fator, através da estimativa estandardizada, é possível afirmar que as variáveis apresentam uma relação significativa bastante elevada com o fator, existindo uma forte relação direta entre si.

Para terminar a análise fatorial confirmatória, é apresentada a estrutura fatorial global, iniciando a discussão com os valores das estimativas estandardizadas (figura 1). 


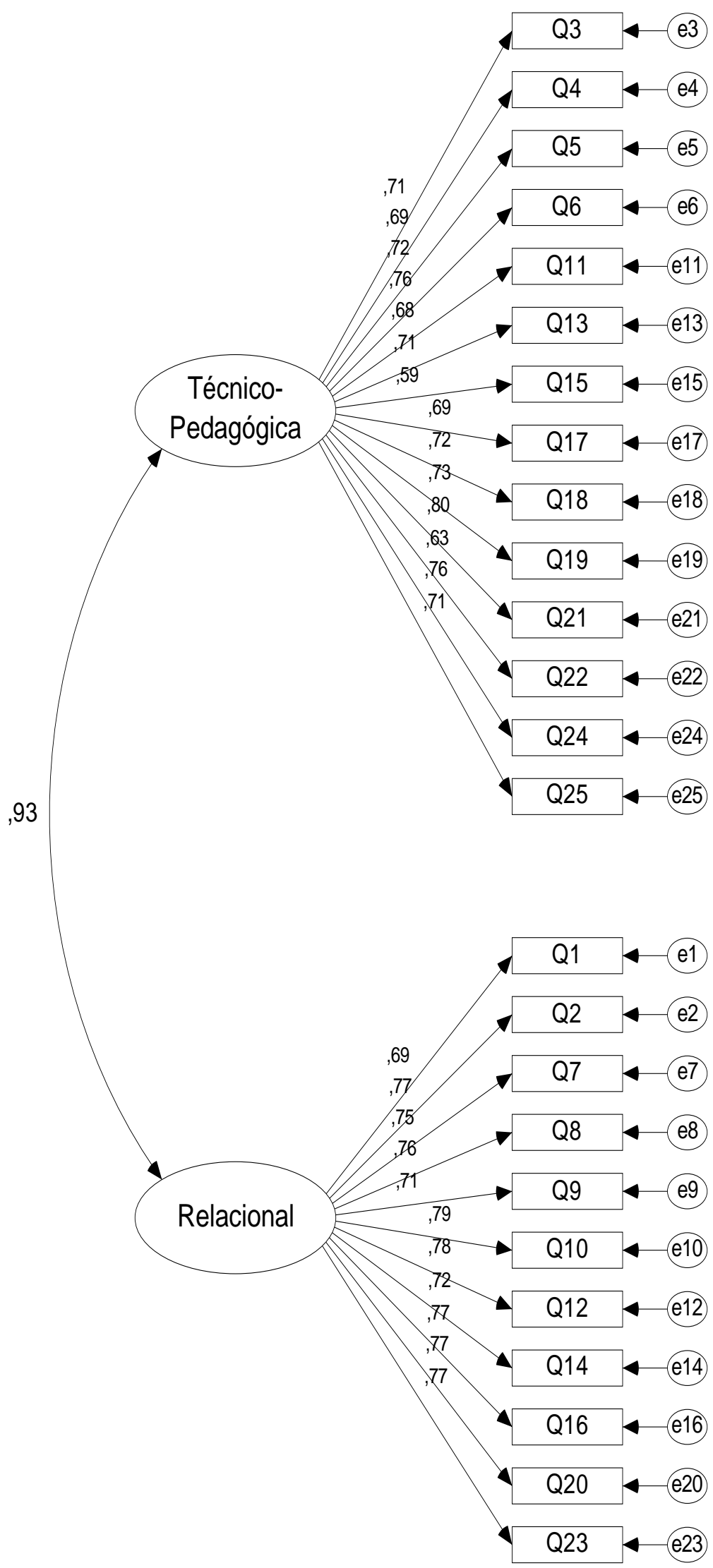

Figura 1. Estimativas estandardizadas da estrutura fatorial global 
Existe validade convergente de ambos os construtos, isto é, de ambos os fatores, pois as saturações fatoriais são elevadas e significativas $(t>1.96)$ (tabela 6).

Tabela 6. Saturações fatoriais estandardizadas da estrutura fatorial global

\begin{tabular}{|c|c|c|c|c|}
\hline Dimensão & Variável & Saturações estandardizadas & Erro padrão & Teste $t^{*}$ \\
\hline \multirow{11}{*}{$\begin{array}{l}\text { Fator } 1 \\
\text { Qualidade } \\
\text { Relacional }\end{array}$} & Q1 & 0.689 & 0.035 & 22.328 \\
\hline & Q2 & 0.767 & 0.036 & 25.362 \\
\hline & Q7 & 0.749 & 0.037 & 24.655 \\
\hline & Q8 & 0.758 & 0.039 & 24.986 \\
\hline & Q9 & 0.710 & 0.044 & 23.137 \\
\hline & Q10 & 0.785 & 0.036 & 26.065 \\
\hline & Q12 & 0.784 & 0.032 & 26.024 \\
\hline & Q14 & 0.715 & 0.037 & 23.333 \\
\hline & Q16 & 0.770 & 0.032 & 25.478 \\
\hline & Q20 & 0.769 & 0.033 & 25.447 \\
\hline & Q23 & 0.766 & & \\
\hline \multirow{14}{*}{$\begin{array}{l}\text { Fator } 2 \\
\text { Qualidade } \\
\text { Técnico- } \\
\text {-pedagógica }\end{array}$} & Q3 & 0.715 & 0.045 & 21.683 \\
\hline & Q4 & 0.694 & 0.046 & 21.045 \\
\hline & Q5 & 0.723 & 0.041 & 21.931 \\
\hline & Q6 & 0.763 & 0.044 & 23.159 \\
\hline & Q11 & 0.676 & 0.043 & 20.491 \\
\hline & Q13 & 0.706 & 0.050 & 21.419 \\
\hline & Q15 & 0.586 & 0.045 & 17.750 \\
\hline & Q17 & 0.695 & 0.047 & 21.076 \\
\hline & Q18 & 0.717 & 0.049 & 21.763 \\
\hline & Q19 & 0.728 & 0.046 & 22.103 \\
\hline & Q21 & 0.796 & 0.041 & 24.178 \\
\hline & Q22 & 0.626 & 0.045 & 18.959 \\
\hline & Q24 & 0.758 & 0.043 & 23.005 \\
\hline & Q25 & 0.713 & & \\
\hline Fator 2 & Fator 1 & Saturações estandardizadas & Erro padrão & Teste $t^{*}$ \\
\hline $\begin{array}{l}\text { Qualidade } \\
\text { Técnico-pedagógica }\end{array}$ & $\begin{array}{l}\text { Qualidade } \\
\text { Relacional }\end{array}$ & 0.928 & 0.026 & 15.574 \\
\hline
\end{tabular}

$* p$ value $<0,001$.

A fiabilidade é verificada em cada construto, resultante da averiguação da consistência interna e da fiabilidade composta, as quais apresentam valores superiores a 0.90. A variância extraída é para todos os fatores e para a escala superior ao mínimo exigido (0.50). As medidas obtidas indicam um bom ajustamento global do modelo aos dados, se considerarmos o CFI (0.916), RMSEA (0.071) e NFI (0.900). No entanto, não indicam um bom ajustamento, se tivermos em consideração o $X^{2}(1600.334 ; p<0.001)$, sendo o seu valor afetado pelo tamanho da amostra e pelo não cumprimento do pressuposto da normalidade. Ao analisar a relação entre variáveis e fatores, verifica-se que a mesma é significativa, existindo uma forte relação direta entre os itens e os fatores extraídos.

\section{DISCUSSÃO}

Considerando os objetivos estipulados - construir e validar um questionário que permita avaliar a qualidade do instrutor em atividades de grupo de fitness - cumprimos o processo metodológico recomendado na literatura consultada (Blunch, 2008; Bryant \& Yarnold, 2000; Byrne, 2001; Hill \& Hill 2002; Maroco, 2010; Moreira, 2009; Munro, 2005; Pereira, 2004; Pestana \& Gageiro, 2008; Tuckman, 2005) relativamente às diferentes fases de construção do instrumento: estudo preliminar 1, estudo preliminar 2 (pré-teste), revisão por experts, estudo piloto, análise fatorial exploratória e análise fatorial confirmatória.

0 questionário teve por base o modelo teórico desenvolvido no estudo preliminar, no qual a qualidade do instrutor era avaliada através de 25 categorias e 4 dimensões (Qualidade Profissional, Qualidade Relacional, Qualidade Técnica e Qualidade Pedagógica) (Campos, 2015). Após o cumprimento das várias fases associadas ao processo de construção, a estrutura fatorial demonstrou algumas fragilidades, tendo o questionário de ser revisto e reformulado de acordo com uma nova estrutura, ligeiramente diferente da inicialmente utilizada (utilizaram-se apenas as 25 categorias, não definindo previamente as dimensões em que estavam agrupadas). Após a análise fatorial exploratória (fase 5) e análise fatorial confirmatória (fase 6), é possível verificar a existência de uma estrutura fatorial aceitável, apesar de algumas das suas limitações, constituída por 2 fatores correlacionados (figura 1): Qualidade 
Relacional (11 itens) e Qualidade Técnico-pedagógica (14 itens). Este modelo, comparativamente com a análise independente do fator 1 (Qualidade Relacional) e do fator 2 (Qualidade Técnico-pedagógica), apresenta índices de ajustamento mais satisfatórios, nomeadamente pelo valor de RMSEA obtido no fator Qualidade Relacional $(0,086)$.

Uma das limitações da estrutura fatorial definida, ao nível da análise fatorial exploratória, é o elevado valor de consistência interna da escala total (0.967) e do fator Qualidade Técnico-pedagógica $(0,953)$. Tal como referido na apresentação dos resultados, estes valores de alta fiabilidade remetem-nos para a possível redundância e fraca discriminação dos itens. Considerando o requisito de cada item contribuir de forma inédita para a total abrangência do construto avaliado, importa que, em termos futuros, seja considerada a reformulação de alguns itens para que esta condicionante seja minimizada. Outra limitação, pela análise fatorial confirmatória, foi o pressuposto da normalidade. Os índices de normalidade, bem como do $X^{2}$, sofreram influência pelo tamanho da amostra. Relativamente ao cumprimento do pressuposto da normalidade, a importância diminui com o aumento da dimensão da amostra. Alguns autores referem que, para amostras grandes, não é necessário o cumprimento do pressuposto da normalidade (Murteira, Ribeiro, Silva \& Pimenta, 2001).

Confrontando os resultados com a literatura consultada (Batista et al., 2008; Cloes et al., 2001; González, Erquicia, \& González, 2005; Papadimitriou \& Karteroliotis, 2000; Wininger, 2002), constatamos que ambas as dimensões (Qualidade Relacional e Qualidade Técnico-pedagógica) foram indicadas como associadas à qualidade do instrutor em atividades de grupo de fitness. 0 mesmo é possível afirmar em relação às categorias, as quais na sua grande maioria foram indicadas, adotando igual denominação ou denominação idêntica, mas com significado semelhante.

Concluindo, após apresentação, análise e discussão dos resultados, é importante refletir sobre a importância do presente trabalho. Mesmo considerando a estrutura fatorial definida como válida, a mesma apresenta algumas limitações, as quais devem ser consideradas em investigações futuras com o objetivo de apresentar uma estrutura fatorial com índices de fiabilidade e validade superiores (tendo como base de trabalho o questionário QIF-AG). Tal instrumento irá permitir, por exemplo: aos proprietários e diretores técnicos de um ginásio, ter indicações acerca do trabalho desenvolvido pelos funcionários (instrutores) e intervir de forma a melhorar os indicadores de qualidade menos positivos; ou aos instrutores, retificar e/ou orientar a sua intervenção profissional de acordo com a perceção dos seus praticantes, adequando o serviço (atividades de grupo de fitness) de acordo com a opinião dos mesmos. Estes exemplos remetem-nos para o problema inicialmente apresentado, focado na necessidade de os ginásios implementarem estratégias para diminuição do abandono e/ou aumento da fidelização (Franco et al., 2008). Sabendo que a qualidade percebida antecede a satisfação e que, a esta, está associada a fidelização (Fernandéz et al., 2012; Pedragosa \& Correia, 2009), este poderá ser um instrumento bastante útil de forma a melhorar a qualidade do serviço prestado pelos instrutores no âmbito das atividades de grupo de fitness.

\section{Referências}

Almeida, L,. \& Freire, T. (2003). Metodologia da investigação em psicologia e educação (3a ed.). Braga: Psiquilíbrios.

Arbuckle, J., \& Wothke, W. (1999). AMOS 4.0. Users guide. Chicago: Smallwaters.

Baker, D., \& Crompton, J. (2000). Quality, satisfaction and behavioral intentions. Annals of Tourism Research, 27(3), 785-804. doi:10.1016/S0160-7383(99)00108-5

Batista, P., Graça, A., \& Matos, Z. (2008). Termos e características associadas à competência. Estudo comparativo de profissionais do desporto que exercem a sua atividade profissional em diferentes contextos de prática desportiva. Revista Portuguesa de Ciências do Desporto, 8(3), 377-395.

Blunch, N. (2008). Introduction to structural equation modeling using SPSS and AMOS. Thousand Oaks: SAGE.

Bryant, F., \& Yarnold, P. (2000). Principal-components analysis and exploratory and confirmatory factor analysis. In L. Grimm, \& P. Yarnold (Eds.), Reading and understanding multivariate statistics (pp. 99136). Washington DC: APA.

Byrne, B. (2001). Structural equation modeling with AMOS. Basic concepts, applications and programming (2nd ed.). New Jersey: Lawrence Erlbaum Associates.

Campos, F. (2015). A qualidade do instrutor em atividades de grupo de fitness. Tese de Doutoramento não publicada, Universidade de Trás-os-Montes e Alto Douro (UTAD) - Vila Real.

Cloes, M., Laraki, N., Zatta, S., \& Piéron, M. (2001). Identification des critères associés à la qualité des instructeurs d'aérobic. Presented at the colloque "L'intervention dans le domaine des activités physiques et sportives", Grenoble - Switzerland.

DeVellis, R. (2003). Scale development (2nd ed.). Thousand Oaks: SAGE. 
Fernandéz, J., Carrión, G., \& Ruíz, D. (2012). La satisfacción de clientes y su relación con la percepción de calidad en centro de fitness: utilización de escala CALIDFIT. Revista de Psicología del Deporte, 21(2), 309-319.

Francis, L., \& Seibert, R. (2000). Teaching a group exercise class. In D. Green (Ed.), Group fitness instructor manual (pp. 179-204). San Diego: ACE.

Franco, S., Pereira, L., \& Simões, V. (2008). Dropout motives in exercise. Presented at the Congress of European College of Sport Science, Estoril - Portugal.

Gillham, B. (2007). Developing a Questionnaire (2nd ed.). New York: Continuum.

González, I., Erquicia, B., \& González, S. (2005). Manual de aeróbic y step. Barcelona: Paidotribo.

Hill, M., \& Hill, A. (2002). Investigação por questionário. Lisboa: Sílabo.

Maroco, J. (2007). Análise estatística com utilização do SPSS (3a ed.). Lisboa: Sílabo.

Maroco, J. (2010). Análise estatística com PASW statistics. Lisboa: Report Number.

Moreira, J. (2009). Questionários: teoria e prática. Coimbra: Almedina.

Murray, D., \& Howat, G. (2002). The relationships among service quality, value, satisfaction, and future intentions of customer at an australian sports and leisure centre. Sport Management Review, 5(1), 25-43. doi:10.1016/S1441-3523(02)70060-0

Munro, B. (2005). Statistical methods for health care research (5th ed.). Philadelphia: Lippincott Williams \& Wilkins.

Murteira, B., Ribeiro, C., Silva, J., \& Pimenta, C. (2001). Introdução à estatística. Lisboa: McGraw-Hill.

Nuviala, A., Pérez-Ordás, R., Osuna, M., Grao-Cruces, A., Nuviala, R., \& Jurado, J. (2012). Calidad, satisfacción y valor percibido de los usuarios de un servicio deportivo público. Revista Movimento, 18(4), 11-32.

Papadimitriou, D., \& Karteroliotis, K. (2000). The service quality expectations in private sport and fitness centers: a reexamination of the factor structure. Sport Marketing Quarterly, 9(3), 157-164.

Pedragosa, V., \& Correia, A. (2009). Expectations, satisfaction and loyalty in health and fitness clubs. International Journal of Sport Management and Marketing, 5(4), 450-464. doi:http://dx.doi.org/10.1504/IJSMM.2009.023371

Pereira, A. (2004). Guia prático de utilização do SPSS. Análise de dados para ciências sociais e psicologia (5a ed.). Lisboa: Sílabo.

Pestana, M., \& Gageiro, J. (2008). Análise de dados para ciências sociais. A complementaridade do SPSS (5a ed.). Lisboa: Sílabo.

Pett, M., Lackey, N., \& Sullivan, J. (2003). Making sense of fator analysis. Thousand Oaks: SAGE.

Tabachnik, B., \& Fidell, L. (2006). Using multivariate statistics (5th ed.). Boston: Pearson Education.

Wininger, S. (2002). Instructors and classroom characteristics associated with exercise enjoyment by females. Perceptual and Motor Skills, 94(2), 395-398. doi:10.2466/pms.2002.94.2.395

$\begin{array}{ll}\text { Historial do } & \text { artigo } \\ \text { Recebido } & 15 / 07 / 2015 \\ \text { Aceite } & 09 / 05 / 2016 \\ \text { Publicado } & 06 / 2016\end{array}$


A qualidade do instrutor de atividades de grupo de fitness 